\title{
Early Risk Indicator of Chemical Dependence: A Comparison Between Emergency and Non-emergency Medicine Residents and Specialists
}

\author{
Maryam Bahreini ${ }^{(\mathbb{D}}{ }^{1}$, Amirhossein Jahanshir ${ }^{1}$, Mehran Sotoodehnia ${ }^{1}$ and Fatemeh Rasooli ${ }^{1, *}$ \\ ${ }^{1}$ Department of Emergency Medicine, Tehran University of Medical Sciences, Tehran, Iran \\ "Corresponding author: Department of Emergency Medicine, Tehran University of Medical Sciences, Tehran, Iran. Email: fa.rasooli2@gmail.com
}

Received 2020 June 25; Revised 2021 March 05; Accepted 2021 April 04.

Keywords: Medical Students, Substance-Related Disorders, Addictive Behavior, Abuse

\section{Dear editor,}

The prevalence of misused substances among physicians during their lifetime has been estimated to be $10-15 \%$, and the rate of drug abuse and addiction is 5 times higher among physicians than general population (1). There are many theories suggesting the etiology of chemical dependence, but it is not limited to genetic, psychological, work exposures and difficulties, and family conditions (2). Sensation seeking (SS) is a personality trait that has been proved to be related to the high-risk behaviors in physical, social, legal, and financial domains $(3,4)$. Sensation seeking behavior is known as a vulnerability factor and a predictor of poorer prognosis in addiction associated with multiple substance use, more functional impairment, and more impaired decision-making $(5,6)$.

We used Zuckerman's sensation seeking scale (ZSSS) to predict the risk of substance use tendencies. It was translated to the native language in the country (Persian in Iran) (5) obtained acceptable statistical characteristics, the face and content validity and reliability. Data were gathered through convenience sampling in all 24/7 clinical shifts (30 shifts) in four university hospitals of two universities in the capital city of the country among consented participants. The shifts consisted of 24 hours part-time shifts for non-EM residents and 12 hour full-time shifts for EM residents and specialists in the emergency departments with a nearly similar method of the nationally-applied residency curriculum. The study was performed in three phases: initially, the first-year emergency and non-emergency residents were compared. Non-EM residents were medical and surgery PG-Y 1 residents. Residents of anesthesiology and psychiatry were excluded due to the evidence for the probability of risky behavior in previous studies 6 . Besides, vari- ous levels of emergency medicine residents (PG-Y1-3) were included. Moreover, we compared emergency medicine specialists with residents.

Overall, 90 subjects, including 71 residents (35 EM and 36 Non-EM residents) and 19 emergency medicine specialists were eager to fill the questionnaires, and 3 physicians were not willing to enter the study. The participants aged 25 - 47 years (mean $\pm \mathrm{SD}=32.97 \pm 5.34$ ) and mostly were 26 - 35 years old. Nearly 52.36 \% of total and 51.4 \% of EM residents were married and no one was divorced. About $25.5 \%$ of the agents experienced parenthood having 1 - 2 children. Also, $11.1 \%$ declared smoking as a habit. Overall, $65.50 \%$ of the participants acquired high scores (5 or more). The detailed demographic results of this study are presented in Table 1 . The scores of ZSSS ranged from zero to 13 (mean $\pm \mathrm{SD}=5.41 \pm 2.48$ ). No significant difference was detected between the civil status, parenthood, or smoking habits of the three study groups. No significant difference was found between EM and non-EM residents in terms of ZSSS (P-value $=0.761$ ) between EM residents and specialists $(P$-value $=0.322)$ and also between EM residents of PG-Y 2, 3 and the new residents of EM (P-value $=0.354$ ), reported in Table 2 . The odds ratio of the sensation seeking risk between EM and non-EM residents was 1.68 (95\% CI 0.69 - 4.40) and was measured to be 2.35 (95 \% CI 0.69 - 7.97) between EM residents and specialists. In our study, we could not find any significant difference between sensation seeking behavior of EM and Non-EM residents and emergency medicine specialists, implying that the level of stress was not increased with higher responsibility and level of training in EM residents. The participants showed no difference in sensation seeking behavior in terms of gender. We need to know more about the 
risk factors of chemical dependence among physicians. Recently, Werb et al. found that sensation seeking is independently associated with substance use and suggested using SSS as a screening test among street youth (7). Gold et al. mentioned that stress and access to drugs might drastically increase the risk of addiction in the workplace (3). Some physician-specific risk factors have been described for substance use, including cigarette use more than one pack-year, high stress or long work hours, history of several marriages, multitask jobs, especially in multiple communities, occupational access to controlled substances, practice in emergency medicine, anesthesiology or psychiatry, and self-medicating or self-prescribing behavior (6). Another study mentioned that substance use showed slight, but non-significant higher rates in emergency medicine, psychiatry, and anesthesiology specialists, and this rate increased in the residency period (6). Furthermore, men and married students were more susceptible than women, probably due to the tendency to seek new experiences and marital conflicts, respectively. Although the workplace seems to be a crucial factor, other underlying factors may be more considerable.

In conclusion, lack of significant difference between sensation seeking behavior of EM and non-EM residents and emergency specialists may indicate that the sensation seeking behavior alone is not probably a major risk factor for higher rates of chemical substance use among emergency physicians.

Limitations: Larger sample sizes permit to empower subgroup analysis and assess and control other leading factors, such as baseline stress and working and educational environment. Furthermore, the level of baseline stress of the participants can be assessed.

\section{Footnotes}

Authors' Contribution: Maryam Bahreini, Amirhossein Jahanshir, and Fatemeh Rasooli designed the trial. Maryam
Bahreini collected data and prepared them for analysis. Amirhossein Jahanshir and Maryam Bahreini analyzed the data. Maryam Bahreini, Fatemeh Rasooli, Mehran Sotoodehnia, and Amirhossein Jahanshir Reviewed the article and submitted it to the journal. All the authors critically appraised and approved the manuscript.

Conflict of Interests: There was no conflict of interest.

Ethical Approval: This study was approved by the institutional review board of the University of Medical Sciences and performed in accordance with ethical standards of the Declaration of Helsinki (Code: 1398.206).

Funding/Support: The research did not receive any funding.

\section{References}

1. Merlo LJ, Trejo-Lopez J, Conwell T, Rivenbark J. Patterns of substance use initiation among healthcare professionals in recovery. Am J Addict. 2013;22(6):605-12. doi: 10.1111/j.1521-0391.2013.12017.x. [PubMed: 24131169].

2. Gold MS, Byars JA, Frost-Pineda K. Occupational exposure and addictions for physicians: case studies and theoretical implications. Psychiatr Clin North Am. 2004;27(4):745-53. doi:10.1016/j.psc.2004.07.006. [PubMed: 15550291].

3. Zuckerman M. Manual and research report for the Sensation Seeking Scale (SSS). Delaware: University of Delaware; 1975.

4. Smorti M, Guarnieri S. Sensation seeking, parental bond, and risky driving in adolescence: Some relationships, matter more to girls than boys. SafSci. 2014;70:172-9. doi: 10.1016/j.ssci.2014.05.019.

5. Ortin A, Lake AM, Kleinman M, Gould MS. Sensation seeking as risk factor for suicidal ideation and suicide attempts in adolescence. J Affect Disord. 2012;143(1-3):214-22. doi: 10.1016/j.jad.2012.05.058. [PubMed: 22921521]. [PubMed Central: PMC3501599].

6. Norbury A, Husain M. Sensation-seeking: Dopaminergic modulation and risk for psychopathology. Behav Brain Res. 2015;288:79-93. doi: 10.1016/j.bbr.2015.04.015. [PubMed: 25907745].

7. Werb D, Richardson C, Buxton J, Shoveller J, Wood E, Kerr T. Development of a brief substance use sensation seeking scale: validation and prediction of injection-related behaviors. AIDS Behav. 2015;19(2):35261. doi: 10.1007/s10461-014-0875-z. [PubMed: 25119056]. [PubMed Central: PMC4450886] 


\begin{tabular}{|c|c|c|c|c|c|}
\hline Participant Characteristics & EM Residents & Non-EM Residents & Emergency Specialists & Total & Significance (ANOVA) \\
\hline $\mathbf{N}$ & 35 & 36 & 19 & 90 & \\
\hline Gender, No. (\%) of men & $17(48.6)$ & $24(66.7)$ & $7(36.8)$ & $48(53.3)$ & 0.085 \\
\hline Age $($ Mean \pm SD $)$ & $34.31 \pm 4.75$ & $31.77 \pm 5.12$ & $32.70 \pm 6.33$ & $32.97 \pm 5.34$ & 0.132 \\
\hline Married, No. (\%) & $18(51.4)$ & $21(58.3)$ & $9(47.4)$ & $48(52.36)$ & 0.718 \\
\hline Smoker, No. (\%) & $4(11.4)$ & $6(16.7)$ & $0(0)$ & $10(11.1)$ & 0.788 \\
\hline Number of children, No. (\%) & & & & & 0.542 \\
\hline 0 & $23(65.7)$ & $29(80.6)$ & $15(78.9)$ & $67(74.4)$ & \\
\hline 1 & $8(22.9)$ & $4(11.1)$ & $1(5.3)$ & $13(14.4)$ & \\
\hline 2 & $4(11.4)$ & $3(8.3)$ & $3(15.8)$ & $10(11.1)$ & \\
\hline
\end{tabular}

Abbreviations: EM: emergency physician; N: number; SD: standard deviation; SSS: sensation seeking behavior.

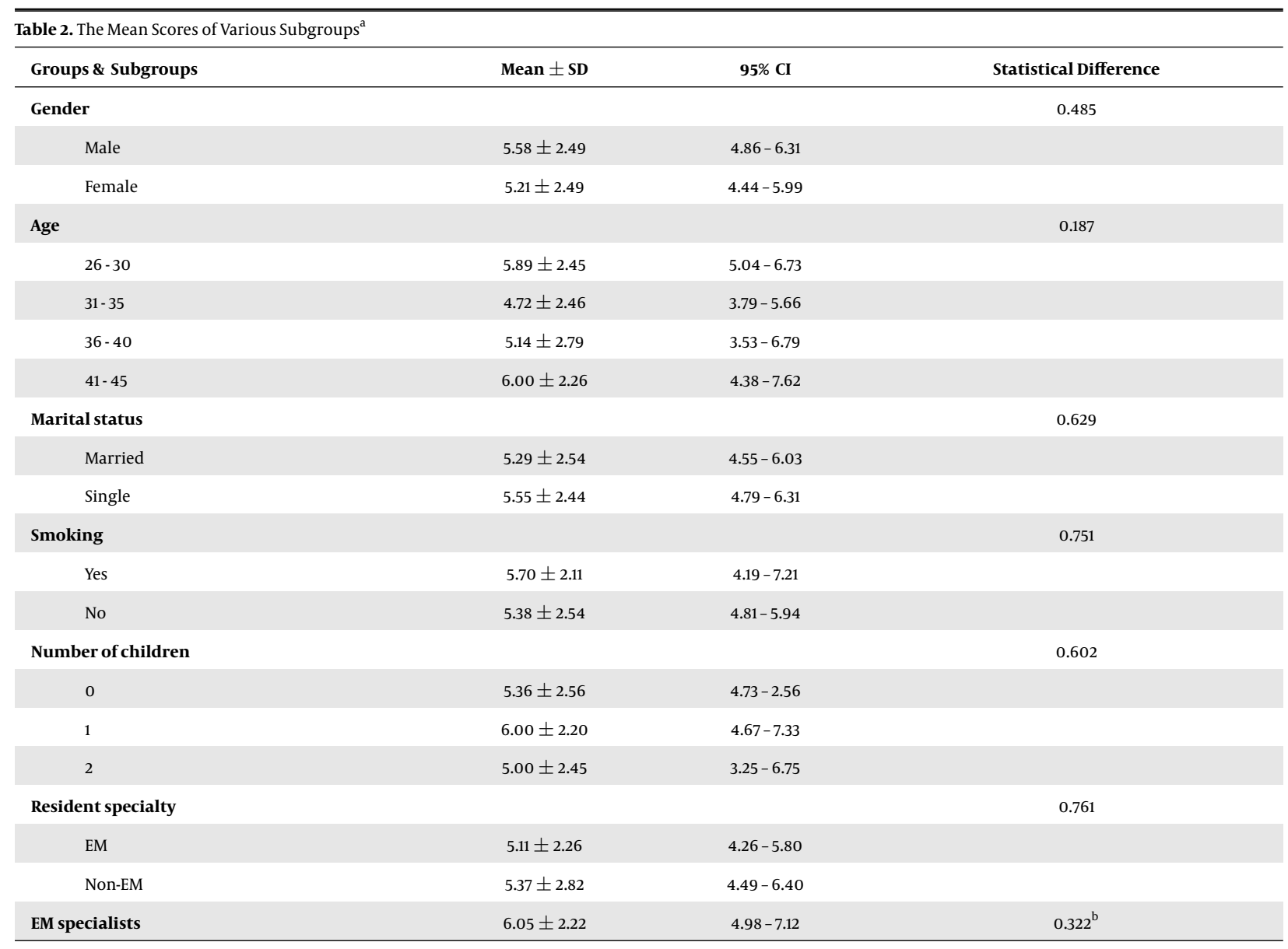

${ }^{\text {a }}$ Only 2 participants were under 25 years old and were not analyzed.

${ }^{\mathrm{b}}$ EM specialists versus EM residents were compared. EM: Emergency medicine; PG-Y: Postgraduate year 